\title{
Directional Transverse Oscillation Vector Flow Estimation
}

\author{
Jensen, Jørgen Arendt
}

Published in:

IEEE Transactions on Ultrasonics, Ferroelectrics and Frequency Control

Link to article, DOI:

10.1109/TUFFC.2017.2710361

Publication date:

2017

Document Version

Peer reviewed version

Link back to DTU Orbit

Citation (APA):

Jensen, J. A. (2017). Directional Transverse Oscillation Vector Flow Estimation. IEEE Transactions on Ultrasonics, Ferroelectrics and Frequency Control, 64(8), 1194-1204.

https://doi.org/10.1109/TUFFC.2017.2710361

\section{General rights}

Copyright and moral rights for the publications made accessible in the public portal are retained by the authors and/or other copyright owners and it is a condition of accessing publications that users recognise and abide by the legal requirements associated with these rights.

- Users may download and print one copy of any publication from the public portal for the purpose of private study or research.

- You may not further distribute the material or use it for any profit-making activity or commercial gain

- You may freely distribute the URL identifying the publication in the public portal

If you believe that this document breaches copyright please contact us providing details, and we will remove access to the work immediately and investigate your claim 


\title{
Directional Transverse Oscillation Vector Flow Estimation
}

\author{
Jørgen Arendt Jensen, IEEE Fellow \\ Center for Fast Ultrasound Imaging, Department of Electrical Engineering, \\ Technical University of Denmark, DK-2800 Lyngby, Denmark
}

\begin{abstract}
A method for estimating vector velocities using transverse oscillation (TO) combined with directional beamforming is presented. In Directional Transverse Oscillation (DTO) a normal focused field is emitted and the received signals are beamformed in the lateral direction transverse to the ultrasound beam to increase the amount of data for vector velocity estimation. The approach is self-calibrating as the lateral oscillation period is estimated from the directional signal through a Fourier transform to yield quantitative velocity results over a large range of depths. The approach was extensively simulated using Field IIpro and implemented on the experimental SARUS scanner in connection with a BK Medical 8820 e convex array transducer. Velocity estimates for DTO are found for beam-to-flow angles of $60^{\circ}, 75^{\circ}$, and $90^{\circ}$, and vessel depths from 24 to $156 \mathrm{~mm}$. Using 16 emissions the Standard Deviation (SD) for angle estimation at depths ranging from 24 to $104 \mathrm{~mm}$ are between $6.01^{\circ}$ and $0.93^{\circ}$ with a mean SD of $2.8^{\circ}$. The mean relative SD for the lateral velocity component is $9.2 \%$ and the mean relative bias $\mathbf{- 3 . 4 \%}$ or 4 times lower than for traditional TO. The approach also works for deeper lying vessels with a slight increase in SD to $15.7 \%$, but a maintained bias of $-3.5 \%$ from 126 to $156 \mathrm{~mm}$. Data for a pulsating flow has also been acquired for 15 cardiac cycles using a CompuFlow 1000 pump. The relative SD was here $7.4 \%$ for a femoral artery waveform.
\end{abstract}

\section{INTRODUCTION}

Measuring volume flow or the degrees of stenosis necessitates the measurement of quantitative velocity values. Often this is performed with spectral velocity imaging, and here a compensation for the beam-to-flow angle has to be performed. This is not possible to perform correctly in many complex vascular geometries for a pulsating flow as the angle will vary as a function of time and space, and there is no single, correct angle. Vector flow imaging is, thus, necessary to make correct assessments. Several authors have presented methods for this including speckle tracking [1], crossed-beams [2], [3], and variations of directional beamforming [4], [5], which all seek to devise quantitative, angle independent measures. Many of these methods have also been combined with fast imaging using synthetic aperture [6] and plane wave imaging [7], [8], [9] and further refined by a number of authors. The dual beam vector velocity method was further investigated by Dunmire et al. [3], Pastorelli et al. [10], and Swillens et al. [11]. Fadnes et al. [12] and Ekroll et al. [9] combined the dual beam approach with the plane wave vector flow imaging to show vector flow images. The plane wave imaging approach has also been extensively investigated by Provost et al. [13] and Bercoff et al. [8] although these are only for the axial velocity component estimation. A comprehensive review of vector flow imaging and the various advantages and disadvantages can be found in [14] and [15].

The velocity vector in a plane can also be estimated using the transverse oscillation (TO) method described in [16], [17], [18], which is a computationally in-expensive method that has been implemented on a commercial platform. A similar approach has also been suggested by Anderson [19] and studied by Sumi [20] and Liebgott et al. [21], [22], [23]. It was also combined with plane wave imaging by Lenge et al. [24]. In TO vector flow imaging (VFI) two beams are formed during receive processing for finding the direction of the flow. In conventional axial velocity estimation this is found using the in-phase and the quadrature signals, which are $90^{\circ}$ phase shifted relative to each other using either a phase delay or a Hilbert transform [25]. In TO the two beams are spatially phase shifted a quarter of the lateral wavelength. This wavelength depends on the emit focus, the receive apodization function, and the interrogation depth. The TO wavelength is predicted from [16], [26]:

$$
\lambda_{x}=\frac{2 \lambda d}{P_{d}}=\frac{2 \lambda d}{N_{d} P_{i}},
$$

where $\lambda$ is the normal axial wavelength, $d$ is the depth, and $P_{d}$ is the distance between the two peaks in the apodization function. The transducer pitch is $P_{i}$, and the number of elements between the peaks is $N_{d}$. The lateral wavelength depends on depth, and therefore has to be calculated for every depth to ensure an unbiased and accurate result. The equation is also only valid in the far-field or at the focus. For a pulsed field this can introduce a significant bias, and optimization has to be employed. This complicates the implementation of the approach [27].

A new method for beamforming transverse to the ultrasound direction is therefore suggested to make the approach selfcalibrating. The beams can be used for velocity estimation, and the lateral oscillation period or wavelength can also be estimated from this data making the method self-calibrating without the need for pre-calculation of the lateral wavelength. This results in a significantly reduced bias in the lateral velocity estimates and in the angle determination over a large range of depths leading to quantitative velocity estimates. These can be used for vector flow imaging or in the quantification of flow parameters in duplex measurements in a single direction as is obtained for a spectral system. The method has previously been presented in a conference paper [28], which this expanded paper is based on. It adds a comprehensive simulation 


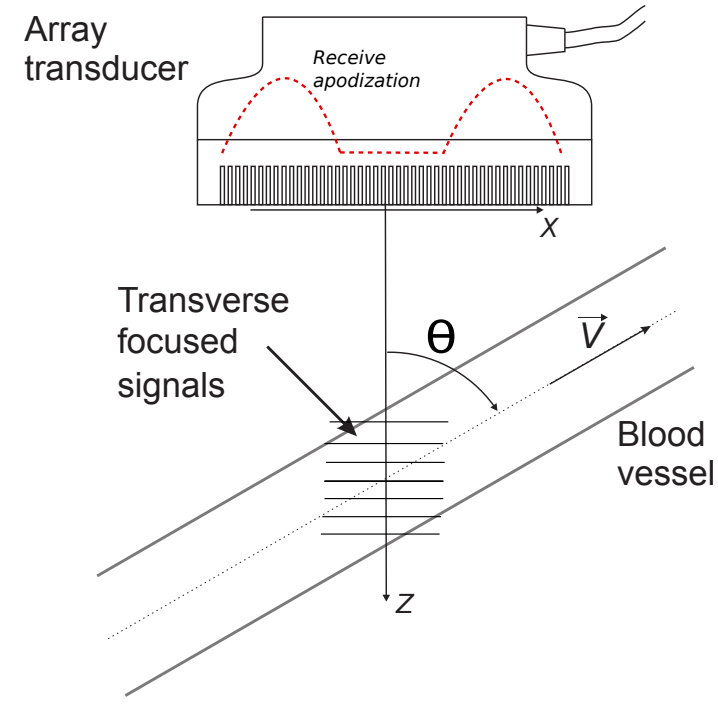

Fig. 1. Focusing of the received signals to generate a signal transverse to the ultrasound direction.

and measurement study along with a detailed derivation of the estimator. The method is described in Section II, and the velocity estimator is derived in Section III. The performance is extensively studied using simulations in Section VI-A and flow rig measurements in Section VI-B. Finally results from a pulsating flow is presented in Section VI-E.

\section{Directional transverse oscillation APPROACH}

The new method beamforms received signals in the lateral direction as shown in Fig. 1. A normal focused field is emitted, and the signals are received on all transducer elements. A beam $x(n, k, i)$ is then focused at the depth of interest in a direction transverse to the ultrasound propagation direction. Here $n$ is the sample index in depth (RF signal), $k$ is the sample number along the directional line, and $i$ is the pulse emission number. Bonnefous [4] suggested focusing a number of parallel beams in receive and then taking data out at one specific depth. Correlating these data from different emissions can then yield the lateral velocity component, when the flow is perpendicular to the ultrasound propagation direction. For other angles the approach breaks down and focusing signals along the flow direction was suggested in [5], [29]. This yields the velocity magnitude, if the proper direction is used as described in [30], [31]. The key idea in this paper is, however, to introduce a lateral oscillation and not to have to focus along the flow, as this necessitates knowing the beam-to-flow angle. The lateral beamformed signals can then be correlated, and the phase shift between two emissions is then proportional to the lateral velocity. The developed estimator below decouples the lateral and axial signals so that the two velocity components can be estimated independently.

A lateral oscillation is obtained, if a receive apodization waveform is employed as shown in Fig. 1 with two peaks separated by a distance $P_{d}$. Two apodization peaks can also be employed for the transmit beam to generate a lateral oscillation with a shorter wavelength.

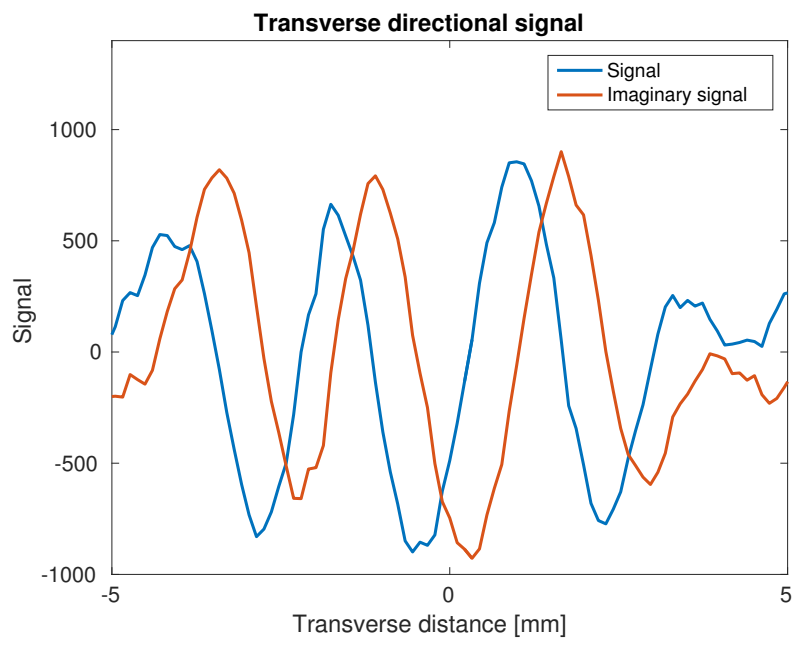

Fig. 2. Directional signal obtained from the focusing. The blue curve is the directional signal and the red curve is the Hilbert transform of the directional signal.

A signal as a function of lateral distance is then beamformed. It is shown as the blue curve in Fig. 2. To perform the velocity estimation, the quadrature signal is also needed [18]. This can be obtained by performing a spatial Hilbert transform of the directional signal:

$$
y(n, k, i)=\mathscr{H}_{k}\{x(n, k, i)\},
$$

where $\mathscr{H}_{k}$ denotes Hilbert transform along the lateral sample direction $k$. It is shown as the red curve in Fig. 2. This yields a combined complex signal with a one-sided spectrum, which directly can be used by the estimator. The mean frequency of one directional signal (blue curve in Fig. 2) yields the lateral oscillation frequency, in the same way as the spectrum of the RF ultrasound signal yields the ultrasound frequency in the $n$ sample direction [32].

An example of the two-dimensional data set used in the approach is illustrated in Fig. 3. Four different oscillations are found in this data set. The emitted ultrasound frequency $f_{0}$ is found along the RF sample direction $n$. The lateral oscillation frequency $f_{l}=1 / \lambda_{x}$ is found along the directional signals direction $k$. Finally, along the emission index $i$ the frequencies are a combination of the axial velocity component $v_{z}$ with a corresponding frequency of $f_{p}=2 v_{z} / \lambda$ and the lateral velocity component $v_{x}$ with a corresponding frequency of $f_{x}=v_{x} / \lambda_{x}$. The method presented here can identify these four frequencies independently, and, thus, compensate the velocity estimates to yield unbiased estimates as a function of depth.

\section{Velocity estimation}

This Section derives a modified estimator developed based on the vector velocity estimator for TO imaging described in [18]. It is modified to include averaging across all samples in the directional signal and along RF samples.

A complex signal is formed from the beamformed directional signal and its spatial Hilbert transform in the direction $k$ as

$$
r_{s q}(n, k, i)=x(n, k, i)+j y(n, k, i) .
$$




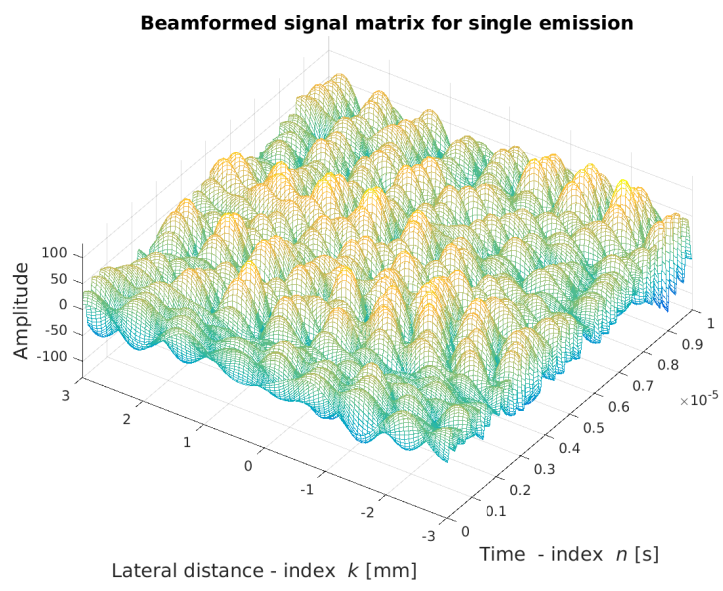

Fig. 3. Two-dimensional data matrix used for the DTO method for a single emission. The first time index $n$ is along the depth direction (Time) and the second index $k$ is along the directional signal transverse to the ultrasound beam (Lateral distance). One 2-D matrix is found for each pulse emission, thus, yielding a 3-D matrix used for the velocity estimation.

The received signals from the transducer are then Hilbert transformed in the temporal direction $n$, and a new directional beamformed signal formed at the same depth for these data. This gives the signal $r_{s q h}(n, k, i)$ as:

$$
r_{s q h}(n, k, i)=\mathscr{H}_{n}\{x(n, k, i)\}+j \mathscr{H}_{n}\{y(n, k, i)\} .
$$

Two new signals are then formed from:

$$
\begin{aligned}
& r_{1}(n, k, i)=r_{s q}(n, k, i)+j r_{s q h}(n, k, i) \\
& r_{2}(n, k, i)=r_{s q}(n, k, i)-j r_{s q h}(n, k, i) .
\end{aligned}
$$

A simple model for the received signal representing all four frequencies found in the received 3-D signal is then [25], [18]:

$$
\begin{aligned}
r_{1}(n, k, i)= & a \cdot \exp \left(j 2 \pi \left[\left(\frac{2 v_{z}}{c} f_{0} i T_{p r f}-f_{0}\left(\frac{n}{f_{s}}+\frac{2 d}{c}\right)\right)\right.\right. \\
& \left.\left.+\left(\frac{v_{x}}{\lambda_{x}} i T_{p r f}-k \frac{\Delta x}{\lambda_{x}}\right)\right]\right) \\
= & a \cdot \exp \left(j \frac{2 \pi}{\lambda}\left(2 v_{z} i T_{p r f}-n \Delta z-2 d\right)\right) \\
& \cdot \exp \left(j \frac{2 \pi}{\lambda_{x}}\left(v_{x} i T_{p r f}-k \Delta x\right)\right)
\end{aligned}
$$

when assuming monochromatic signals. Here $c$ is the speed of sound, $\Delta x$ is the sampling interval along the lateral signal, $a$ is a constant, $f_{s}$ is the temporal sampling frequency, $\Delta z=c / f_{s}$, and $T_{p r f}$ is the time between pulse emissions. The interrogation depth is $d$, and the two frequencies received from the axial and lateral motions are given by:

$$
f_{p}=\frac{2 v_{z}}{c} f_{0}=\frac{2 v_{z}}{\lambda}, \quad f_{x}=\frac{v_{x}}{\lambda_{x}} .
$$

The changes in phase as a function of emission number for the two signals $r_{1}(n, k, i)$ and $r_{2}(n, k, i)$ are [18]:

$$
\begin{aligned}
\frac{\Delta \Theta_{1}}{\Delta i} & =2 \pi T_{p r f}\left(f_{x}+f_{p}\right) \\
\frac{\Delta \Theta_{2}}{\Delta i} & =2 \pi T_{p r f}\left(f_{x}-f_{p}\right) .
\end{aligned}
$$

The transverse velocity can, thus, be found directly from:

$$
v_{x}=\frac{\left(\frac{\Delta \Theta_{1}}{\Delta i}+\frac{\Delta \Theta_{2}}{\Delta i}\right) \lambda_{x}}{2 \pi 2 T_{p r f}}
$$

and the axial velocity from

$$
v_{z}=\frac{\left(\frac{\Delta \Theta_{1}}{\Delta i}-\frac{\Delta \Theta_{2}}{\Delta i}\right) \lambda}{2 \pi 4 T_{p r f}} .
$$

For a complex signal the phase change is determined from the estimated complex autocorrelation of the signal [25], [33]. For the directional signals the autocorrelation function estimates are:

$$
\begin{aligned}
\hat{R}_{1}\left(1, N_{z}\right)= & \frac{1}{\left(N_{e}-1\right) N_{d} N_{s}} \sum_{n=-\left(N_{s}-1\right) / 2}^{\left(N_{s}-1\right) / 2} \sum_{i=0}^{N_{e}-2} \\
& \sum_{k=0}^{N_{d}-1} r_{1}^{*}\left(n+N_{z}, k, i\right) r_{1}\left(n+N_{z}, k, i+1\right),
\end{aligned}
$$

and

$$
\begin{aligned}
\hat{R}_{2}\left(1, N_{z}\right)= & \frac{1}{\left(N_{e}-1\right) N_{d} N_{s}} \sum_{n=-\left(N_{s}-1\right) / 2}^{\left(N_{s}-1\right) / 2} \sum_{i=0}^{N_{e}-2} \\
& \sum_{k=0}^{N_{d}-1} r_{2}^{*}\left(n+N_{z}, k, i\right) r_{2}\left(n+N_{z}, k, i+1\right) .
\end{aligned}
$$

The autocorrelation estimates are, thus, averaged over the number of emissions $N_{e}$, and the number of RF samples $N_{s}$ around the estimation depth sample $N_{z}$ as in [18]. Further averaging over the number of samples in the directional lines $N_{d}$ is used in the new estimator to reduce noise and improve on estimation accuracy.

The velocity estimators for the two velocity components are then:

$$
\begin{aligned}
v_{x}\left(N_{z}\right)= & \frac{\lambda_{x}}{2 \pi 2 T_{p r f}} \arctan \times \\
& \left(\frac{\mathfrak{I}\left\{R_{1 z}(1)\right\} \mathfrak{R}\left\{R_{2 z}(1)\right\}+\mathfrak{I}\left\{R_{2 z}(1)\right\} \mathfrak{R}\left\{R_{1 z}(1)\right\}}{\mathfrak{R}\left\{R_{1 z}(1)\right\} \mathfrak{R}\left\{R_{2 z}(1)\right\}-\mathfrak{I}\left\{R_{1 z}(1)\right\} \mathfrak{I}\left\{R_{2 z}(1)\right\}}\right)
\end{aligned}
$$

and

$$
\begin{aligned}
v_{z}\left(N_{z}\right)= & \frac{\lambda}{2 \pi 4 T_{p r f}} \arctan \times \\
& \left(\frac{\mathfrak{J}\left\{R_{1 z}(1)\right\} \Re\left\{R_{2 z}(1)\right\}-\mathfrak{I}\left\{R_{2 z}(1)\right\} \Re\left\{R_{1 z}(1)\right\}}{\mathfrak{R}\left\{R_{1 z}(1)\right\} \Re\left\{R_{2 z}(1)\right\}+\mathfrak{I}\left\{R_{1 z}(1)\right\} \mathfrak{I}\left\{R_{2 z}(1)\right\}}\right),
\end{aligned}
$$

when using the derivation from [18] to combine the two phase shift estimates into one equation. Here $\mathfrak{I}$ denotes imaginary part, and $\Re$ real part and $R_{1 z}(1)$ corresponds to $R_{1}\left(1, N_{z}\right)$.

The lateral velocity is directly scaled by the lateral wavelength $\lambda_{x}$, which varies as a function of depth. In current TO schemes this is estimated for the various depths, and, thus, has to be predicted either using (1) or found through computer simulations [27] to avoid a bias on the lateral velocity component. The approach here is to use the lateral directional signal to estimate $\lambda_{x}$ from the acquired data. This can be calculated by:

$$
1 / \lambda_{x}(n)=f_{l}(n)=\frac{\sum_{m=-N_{d} / 2-1}^{N_{d} / 2} \frac{m}{N_{d} \Delta x}\left|R_{s q}(n, m, i)\right|^{2}}{\sum_{m=-N_{d} / 2}^{N_{d} / 2}\left|R_{s q}(n, m, i)\right|^{2}}
$$




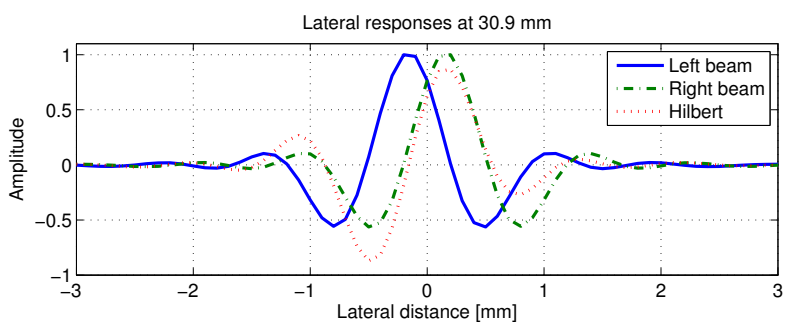

2D Lateral frequency spectrum for combined signal

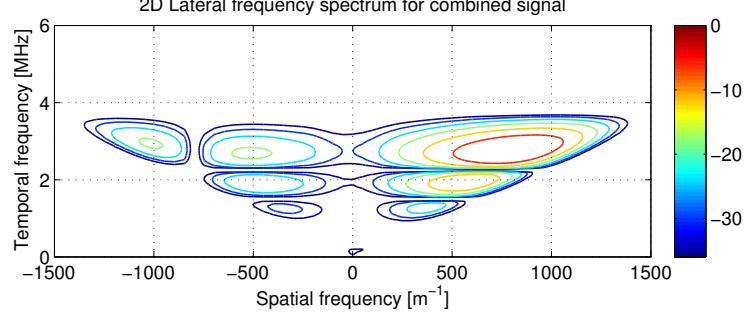

Fig. 4. Lateral response of the TO field at the peak position (top graph) and the corresponding Fourier transform of the complex PSF using the left and right beam. The blue and dashed green lines show the TO signals, and the lower graph shows the corresponding amplitude spectrum. The red line is the Hilbert transform of the blue line, and shows the ideal response for the green dashed line.

where $R_{s q}(n, m, i)$ is the Fourier transform of the complex directional signal $r_{s q}(n, k, i)=x(n, k, i)+j y(n, k, i)$ along the directional signal direction $k$ and at the RF sample index $n$. This makes the approach self calibrating as in [32] for axial velocity estimation and automatically takes diffraction, attenuation, and absorption into account. The lateral wavelength $\lambda_{x}$ is then calculated for the different depths and emissions to yield an unbiased estimate of the transverse velocity. These frequency estimates are improved by averaging over all emissions and over the pulse length as

$$
f_{l}\left(N_{z}\right)=\frac{1}{N_{p}} \sum_{n=N_{z}-N_{p} / 2}^{N_{z}+N_{p} / 2-1} \frac{1}{N_{e}} \sum_{i=0}^{N_{e}-1} \frac{\sum_{m=-N_{d} / 2-1}^{N_{d} / 2} \frac{m}{N_{d} \Delta x}\left|R_{s q}(n, m, i)\right|^{2}}{\sum_{m=-N_{d} / 2}^{N_{d} / 2}\left|R_{s q}(n, m, i)\right|^{2}},
$$

where $N_{p}$ is the number of samples in the ultrasound pulse.

The bias on the axial velocity component is determined by $\lambda$ as this directly scales the velocity estimate [25]. This bias can, thus, be maintained low, if the correct wavelength is employed or estimated from the data as in [34], [35]. In this paper a fixed value directly calculated from the transducer center frequency and the speed of sound in the medium is used.

The beamforming can be performed for a normal focused emission, for synthetic aperture flow imaging, and for plane wave imaging. The transmit and receive apodization function can be changed as a function of depth to obtain the highest possible $f_{l}$. In synthetic aperture and plane wave imaging the combined transmit apodization function can also be manipulated to further increase the lateral oscillation frequency.

\section{DESIGNING DTO FIELDS}

The spatial Hilbert transform yields a one-sided spectrum for the DTO field. This avoids the need for having spatial quadrature ultrasound beams as described in [27]. It is, thus, possible to design the ultrasound field with a more narrow beam without having the spectral leakage into negative spatial frequencies shown in Fig. 4. The top graphs shows the lateral signal at the peak in the TO field for both the left (solid blue curve) and right (dashed green curve) beamformed signal. The Fourier transform of the complex PSF is shown in the lower graph and indicates a high spatial oscillation frequency of $710.0 \mathrm{~m}^{-1}$ corresponding to a wavelength of $1.41 \mathrm{~mm}$ at a depth of $30 \mathrm{~mm}$. The lateral signals nearly form a spatial quadrature after optimization, but the dotted red line shows the ideal response from DTO beamforming. The deviation in the traditional quadrature beamforming gives a spectral leakage ratio of $-14.8 \mathrm{~dB}$ as shown in the lower graph, which can be avoided in DTO beamforming. It is, thus, possible to design DTO fields for a higher spatial resolution and a higher spatial oscillation frequency, and still attain a low bias as the resulting estimates are not affected by spectral leakage. A receive F-number of 0.5 has therefore been selected for DTO beamforming, which is a factor of 4 smaller than previously used in TO beamforming [27].

\section{Simulations, MEASUREMENTS, AND PROCESSING}

The velocity estimation approach has been implemented on the SARUS experimental scanner [36]. A BK Medical 8820 e convex array transducer was employed, and vector flow imaging (VFI) in a single direction was interleaved with a B-mode image. An active aperture of 64 elements was used during transmit for both sequences. The focal point was at 42 $\mathrm{mm}(F \#=2)$ for the B-mode and $105.6 \mathrm{~mm}(F \#=5)$ for VFI in vessels down to $80 \mathrm{~mm}$. A transmit focus of $300 \mathrm{~mm}$ was used for deeper lying vessels, as the transmitted field has to be broad enough to cover the length of the directional line. A single cycle excitation was used for B-mode imaging and four cycles for flow imaging with a center frequency of 3 MHz. The transducer has 192 elements with $\lambda$ pitch and the B-mode image consisted of 129 lines. A $6 \mathrm{~mm}$ radius tube in a circulating flow rig was scanned. The flow was stationary, and a $1.2 \mathrm{~m}$ entrance tube length ensured a parabolic flow. The volume flow was measured by a Danfoss Magnetic flow meter MAG1100 (Danfoss, Nordborg, Denmark) for calculating the peak velocity in the parabolic profile used as a reference.

The volume flow was $106.4 \mathrm{l} / \mathrm{h}$ corresponding to a peak velocity in the vessel of $0.52 \mathrm{~m} / \mathrm{s}$. There was a slight variation among measurements in the pump output, and this was accounted for by measuring the flow rate with the Danfoss meter (stated accuracy of 1\%). The pulse repetition frequency was $2.5 \mathrm{kHz}$ and the beam-to-flow angles were 60,75 , and 90 degrees. The measurement depths were $24,44,64,84$, and $104 \mathrm{~mm}$ from the transducer surface to the vessel center. Measurements were also conducted at 126, 146, and $156 \mathrm{~mm}$. Here the transmit focus was at $300 \mathrm{~mm}$ and the pulse repetition frequency was reduced to $2 \mathrm{kHz}$. Twenty frames were acquired for each situation corresponding to 2,580 emissions or 1.03 and 1.29 seconds of data.

Pulsating flow was also investigated by using a CompuFlow 1000 pump (Shelley Medical Imaging Technologies, Toronto, 


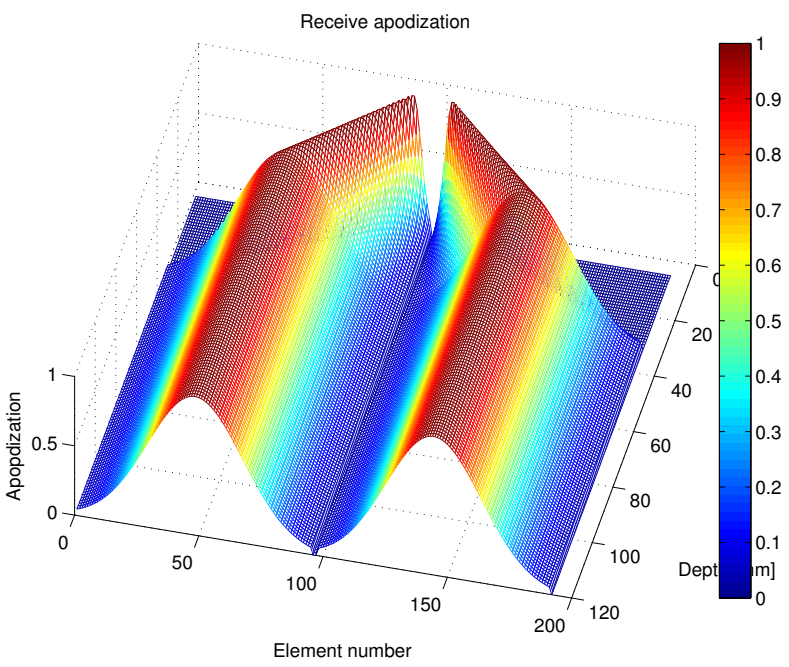

Fig. 5. Dynamic apodization profiles as a function of depth for the receive processing. The distance between peaks and the widths are adjusted according to the receive F-number of 0.5 .

Canada) capable of generating time-varying waveforms. It was connected to a straight tube phantom with an internal diameter of $8 \mathrm{~mm}$ placed $38.2 \mathrm{~mm}$ from the probe. The pump was set to produce a waveform mimicking that of a femoral artery, and of stationary parabolic flow. The duration of one pump cycle was $840 \mathrm{~ms}$ and data for 15 cycles were acquired for 250 frames (12.9 seconds of data).

\section{A. Simulations}

An automatic simulation approach was developed based on the parameters stored by the SARUS system to make them exactly the same as for the measurements. From these the setup and flow were simulated using Field IIpro [37], [38], [39] and the same parameters as for the measured data. The impulse response was measured for the probe and included in the simulation as described in [40]. A wall-less phantom with the same dimensions and flow as for the measurements was simulated. The tube radius was $6 \mathrm{~mm}$, and its lateral length was $36 \mathrm{~mm}$. Ten thousand point scatterers were simulated in the tube, and ten full images were simulated with 129 emissions each. No noise was added to the simulated data to reveal the ideal estimator performance.

\section{B. Processing}

$\mathrm{RF}$ data for all transducer elements were stored for off-line beamforming by the BFT3 beamforming toolbox [41]. Data from simulations and measurements were processed using the same parameters. The beamformation employed a double Gaussian apodization on the receiving aperture, and the Fnumber in receive was 0.5 . The beamformed directional lines had a length of $20 \lambda$ and a sampling interval of $\lambda / 4$. The receiving aperture was expanded as a function of depth to maintain a constant F-number and thereby a constant $\lambda_{x}$ until all 192 elements were used. The resulting apodization profiles are shown in Fig. 5.
Stationary echo canceling was performed by subtracting the mean value from the signals to minimize stationary components giving a cut-off frequency of $f_{p r f} / N$, where $N$ is the number of emissions for the estimate. The estimated correlation functions were averaged over one pulse length for both the axial and lateral velocity components, and the axial component was found using the autocorrelation approach [33] with RF averaging [32]. DTO signals with a length of $\pm 10 \lambda$ centered around the ultrasound beam were beamformed and used for the velocity estimation before using the estimator described in Section III. The lateral oscillation period was estimated continuously as a function of depth using (15) for the same data as used for the velocity estimation. The data were also processed using the method described in [27] for comparison.

The performance of the method is evaluated using the relative bias and relative standard deviation normalized with the peak velocity in the vessel. This is around $0.52 \mathrm{~m} / \mathrm{s}$, and an average is taken across the central $90 \%$ of the vessel to avoid echo canceling effects at the vessel wall.

\section{RESUlts}

These Sections describes the obtained results from the simulations in Section VI-A and for the flow rig measurements in Section VI-B. Section VI-C compares DTO to the TO approach described in [27], and results for deeper lying vessels are described in Section VI-D. Finally results for pulsating flow are described in Section VI-E.

\section{A. DTO Simulations}

Typical velocity profiles for eight emissions are shown in Fig. 6 at a vessel center depth of $44 \mathrm{~mm}$ and at a beamto-flow angle of $90^{\circ}$. The top graph shows the mean of the estimated axial velocity component as the solid blue line and \pm one standard deviation (SD). The lower graph shows the corresponding values for the lateral velocity component. The overall relative standard deviation for $v_{x}$ is $4.49 \%$ with a bias of $3.98 \%$, predominately due to the increased width at the edges of the vessel. The peak velocity that can be estimated is $1.85 \mathrm{~m} / \mathrm{s}$, and the relative values would then be $1.1 \%$ for SD and $1.25 \%$ for the bias. Large deviations in velocities are seen outside the vessel, where the velocity should be zero. For stationary objects the echo canceling filter removes all signal energy as two measurements give exactly the same signal for zero velocity. Thus, only noise is left, and the estimator yields an arbitrary velocity estimate. This is usually handled by having a discriminator that sets velocity estimates to zero, when the residual energy is low compared to the signal before echo canceling [25]. This has not been included here, and the performance of the estimator is evaluated from the central $90 \%$ of the vessel.

The number of emissions used for velocity estimation affects the performance of the approach as shown in Fig. 7. The SD drops from $4.4 \%$ for 8 emissions to $3 \%$ for 64 emissions, and the bias from $4.5 \%$ to $-2 \%$. The effect is small and indicates that acceptable velocity estimates can be attained for only 8 emissions, and the improvement for more 

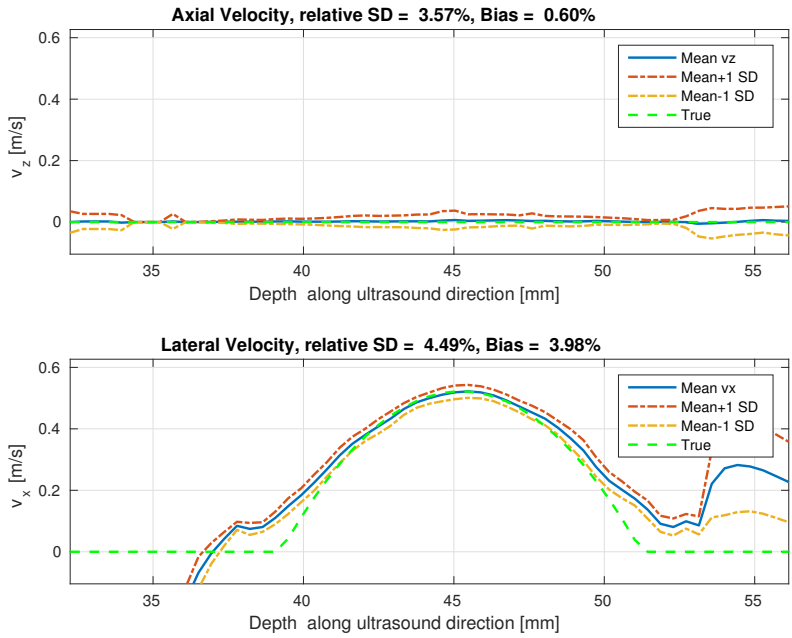

Fig. 6. Mean velocity profile for parabolic simulated flow at a vessel depth of $44 \mathrm{~mm}$ and using 8 emissions for the estimation at a beam to flow angle of $90^{\circ}$.
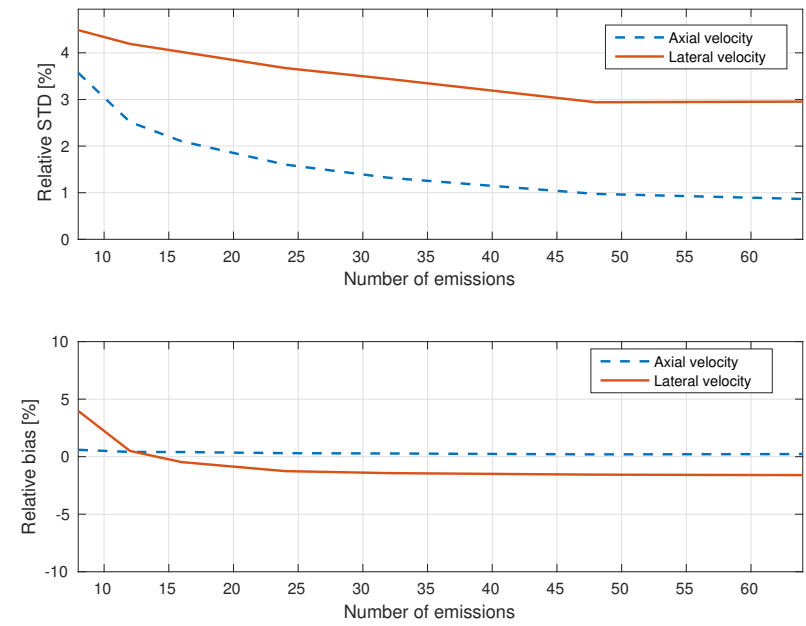

Fig. 7. Standard deviation and bias as a function of number of emissions used in the estimation of the velocity at a vessel depth of $44 \mathrm{~mm}$ at $90^{\circ}$ for simulated data.

emissions is minor and probably due to a slightly better echo canceling. The SD and bias for the axial velocity estimates are roughly lower by a factor between 1.5 and 3 than for the transverse component, which is consistent with findings in previous publications [16], [27].

Similar graphs for a vessel depth of $84 \mathrm{~mm}$ are shown in Fig. 8. The SD is increased to $8.5 \%$ and the bias to $12.6 \%$, which can be reduced by employing 16 or 32 emissions to attain the same level of performance as for a vessel depth of $44 \mathrm{~mm}$.

The SD as a function of depth and angle is shown in Fig. 9. The standard deviation is increasing slightly with depth and also increases, when the angle is decreased from $90^{\circ}$ to $60^{\circ}$. The SD drops with an increasing number of emissions used for the estimation. Similar figures for the bias are shown in Fig. 10 , where it can be seen that the bias and SD is maintained for all depths and angles. For 16 emissions the maximum bias for all situations is $3.4 \%$, the minimum bias is $-8.9 \%$, and
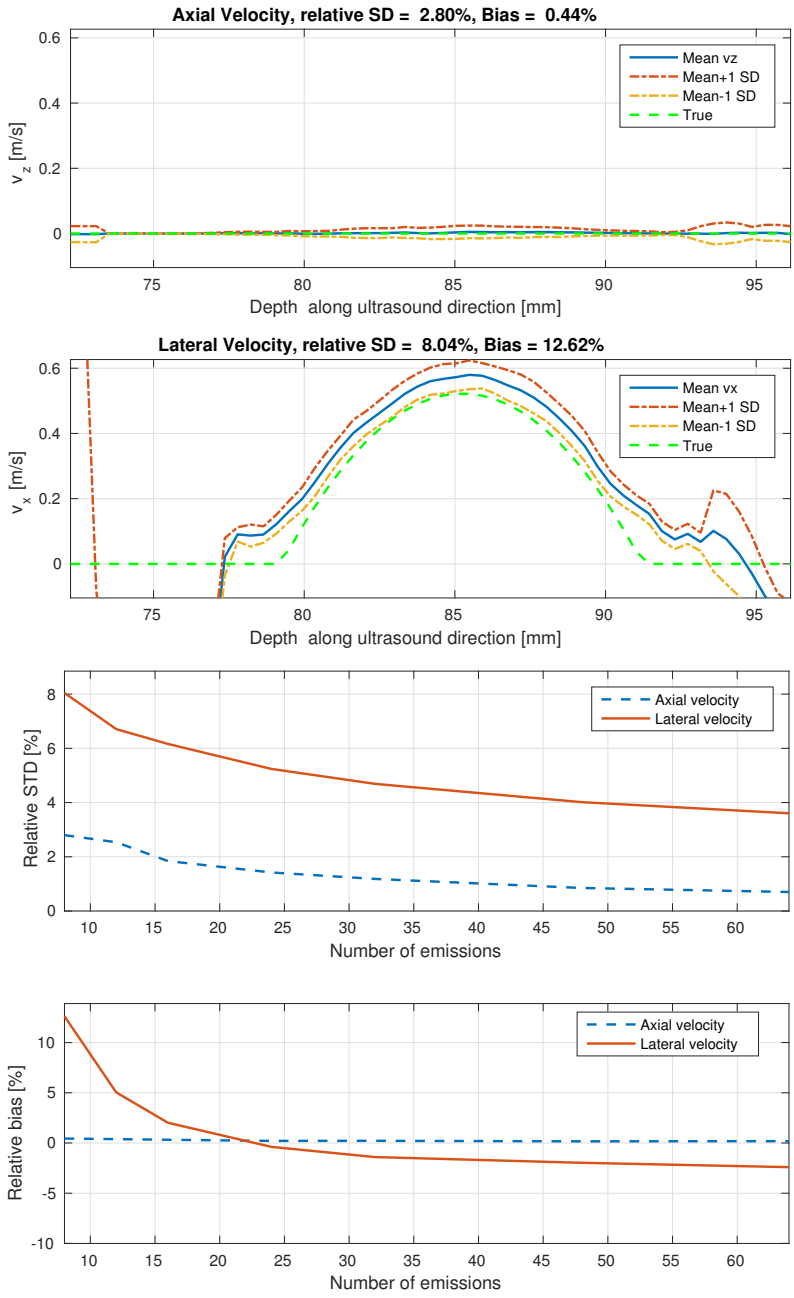

Fig. 8. Mean velocity profile for parabolic simulated flow at a vessel depth of $84 \mathrm{~mm}$ (top graphs) for 8 emissions, and standard deviation and bias as a function of number of emissions (bottom graphs).

the mean SD for all situations is $3.6 \%$ for the lateral velocity component.

Having a precise estimate of both the axial and lateral velocity component makes it possible to estimate the beamto-flow angle. The resulting mean angles \pm one standard deviation are shown in Fig. 11 for 16 emissions. From 24 to $104 \mathrm{~mm}$ the angle is estimated within a maximum SD of $6.0^{\circ}$ with a minimum SD of $1.11^{\circ}$ and an average SD of $2.5^{\circ}$. The angle SD is increasing with decreasing beam-to-flow angle as the axial velocity component increases. Similar trends are seen for other number of emissions, where the angle SD decrease with an increasing number of emissions ( 8 lines: $4.2^{\circ}, 64$ lines: $1.5^{\circ}$.

\section{B. Flow rig measurements}

The performance for the flow rig measurements is shown in Fig. 12 at a vessel center depth of $44 \mathrm{~mm}$ and at a beam-to-flow angle of $90^{\circ}$ and 16 emissions. The top graphs shows the mean of the estimated axial velocity component as the solid blue line and \pm one standard deviation (SD). The lower graph shows the corresponding values for the lateral velocity component. 

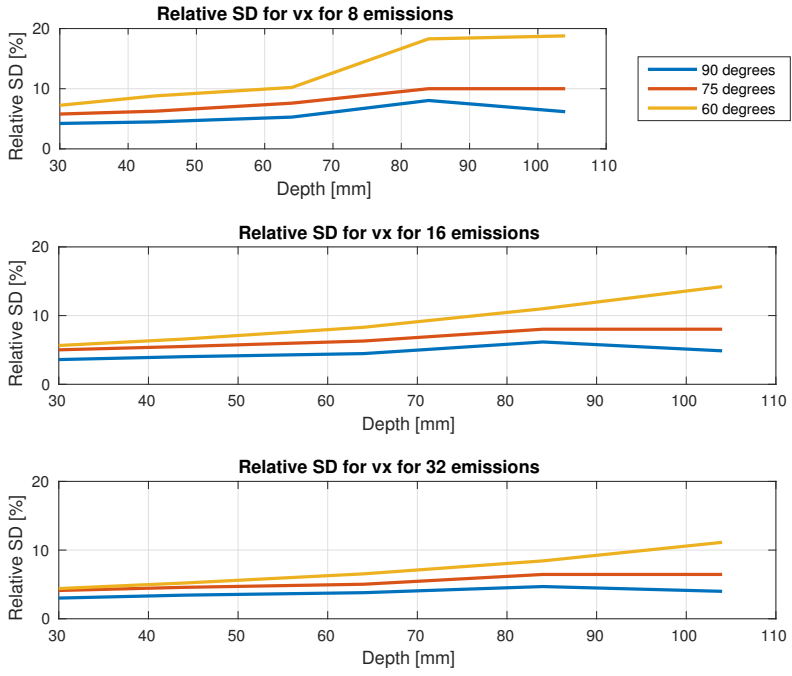

Fig. 9. Relative SD as a function depth and angle for 8 (top), 16 (middle) and 32 emissions (bottom) for the simulated lateral velocity component.
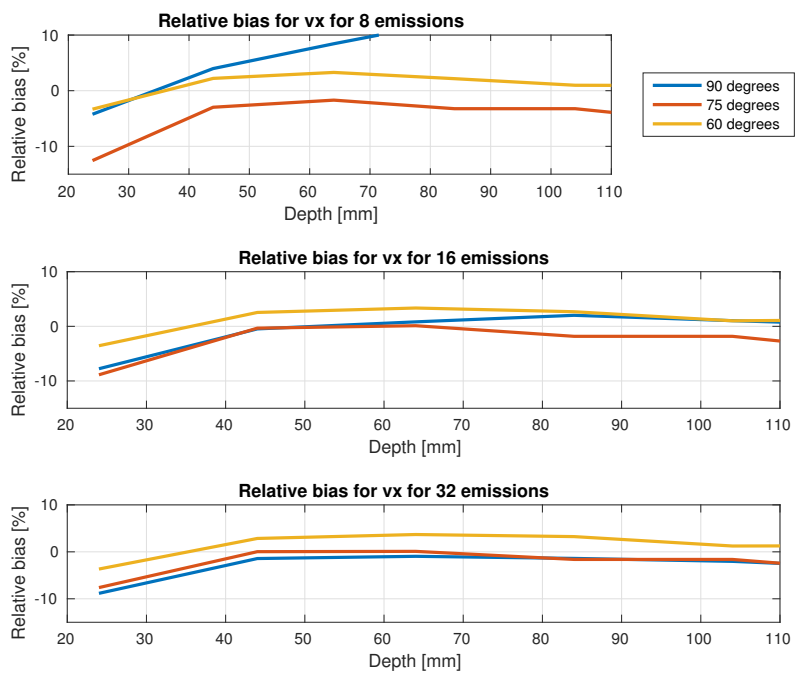

Fig. 10. Relative bias as a function depth and angle for 8 (top), 16 (middle) and 32 emissions (bottom) for the simulated lateral velocity component.

In the measurement setup the angle is not precisely $90^{\circ}$, and the mean estimated angle has therefore been used for the reference waveform. This usually gives a change in angle below 1 degree. The overall relative standard deviation for $v_{x}$ is $6.14 \%$ with a bias of $-3.77 \%$. This is slightly higher than for the simulated flow, where the bias is $-0.47 \%$. This is, however, within the tolerances of the transducer holder, which are around $\pm 1 \mathrm{~mm}$ and $1^{\circ}$. A $1 \mathrm{~mm}$ offset from the vessels' center position will give a bias of $2.8 \%$.

The performance as a function of depth and angle for the measured data is shown in Fig. 13. Overall the performance is slightly worse for the measured data. The standard deviation is slightly higher than for the simulations, and the bias is consistently more negative as shown in Fig. 14. The bias for the lateral velocity component $v_{x}$ for depths from 24 to 104 $\mathrm{mm}$ using 16 emissions spans from $2.7 \%$ to $-11.1 \%$ with a mean bias for all situations of $-3.4 \%$.

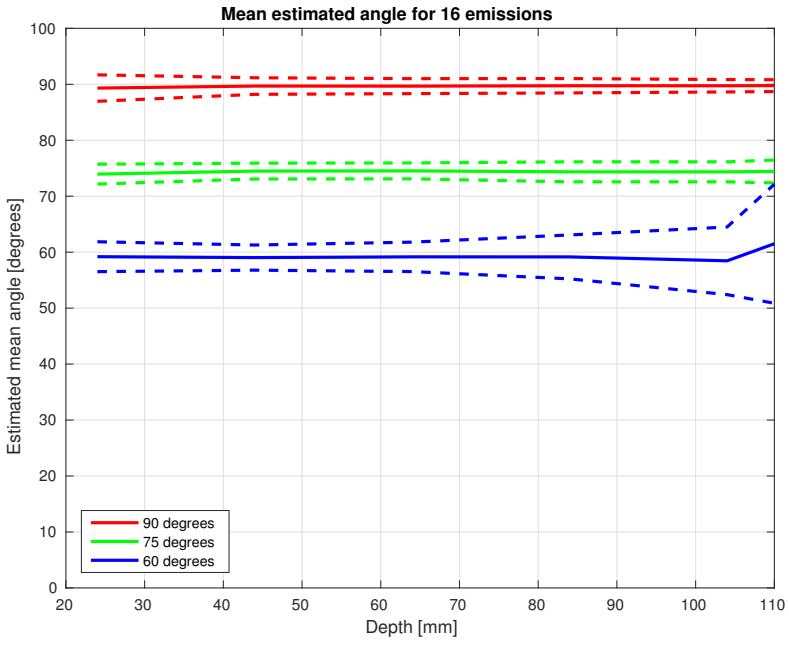

Fig. 11. Estimated angle \pm one standard deviation as a function depth and angle for simulated data and 16 emissions.
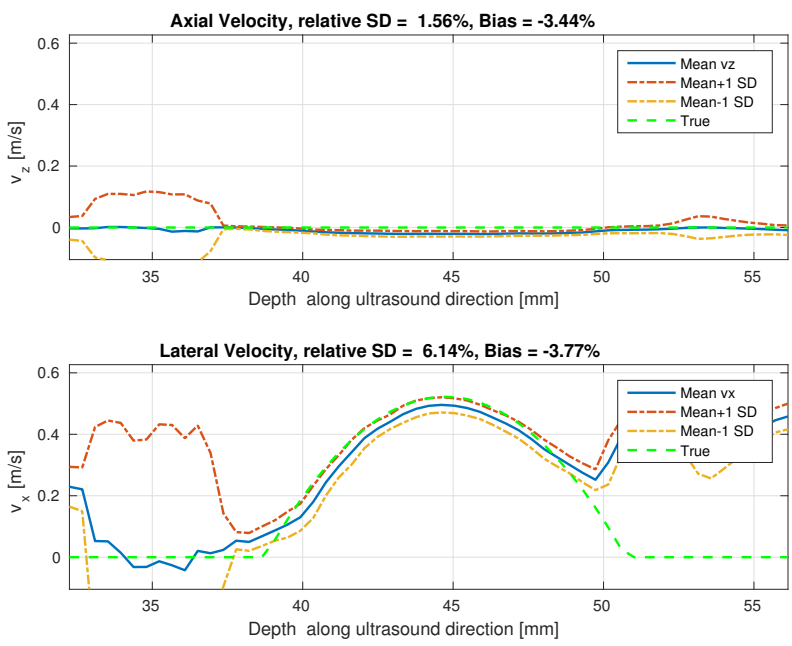

Fig. 12. Mean velocity profile for measured parabolic flow at a vessel depth of $44 \mathrm{~mm}$ and using 16 emissions for the estimation.
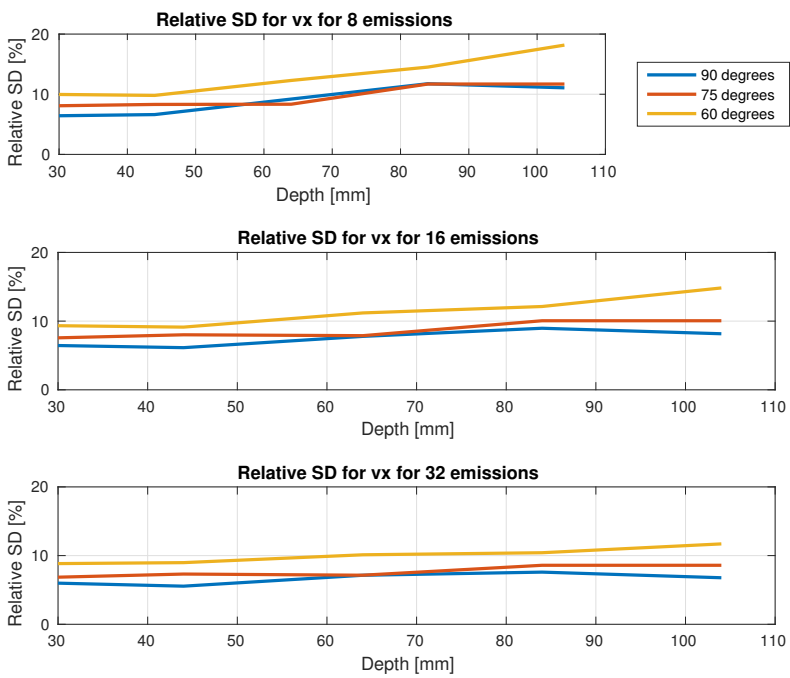

Fig. 13. Measured relative SD as a function depth and angle for 8 (top), 16 (middle) and 32 emissions (bottom). 

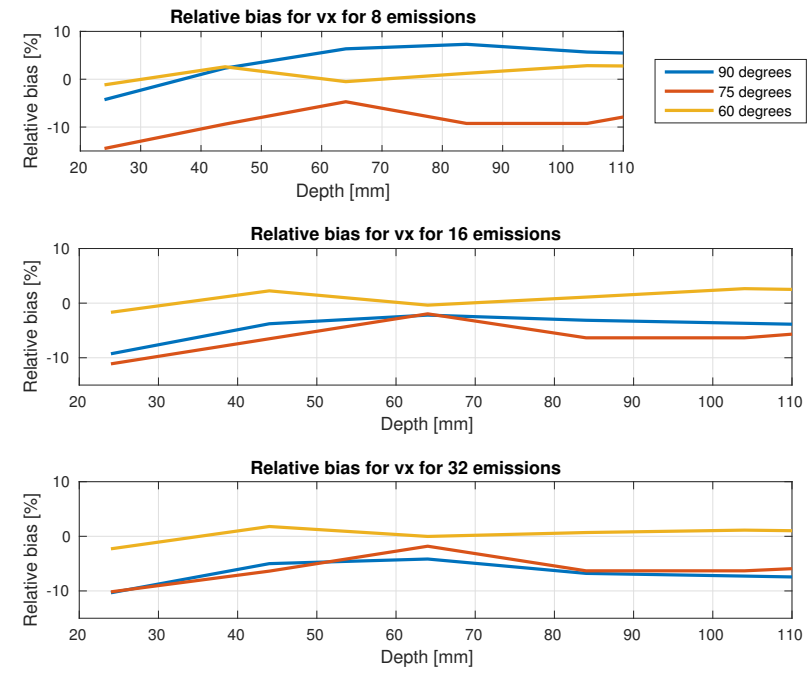

Fig. 14. Measured relative bias as a function depth and angle for 8 (top), 16 (middle) and 32 emissions (bottom) for the lateral velocity component.

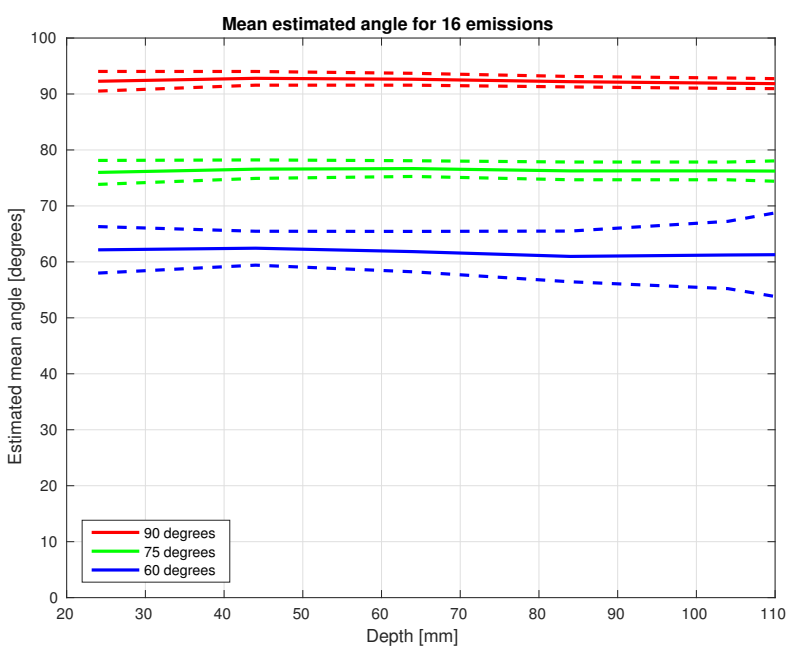

Fig. 15. Estimated angle \pm one standard deviation as a function depth and angle for measured data and 16 emissions.

The resulting mean angles \pm one standard deviation for measured data are shown in Fig. 15 for 16 emissions. From 24 to $104 \mathrm{~mm}$ the angle is estimated within a maximum SD of $6.0^{\circ}$ with a minimum SD of $0.93^{\circ}$ and an average SD of $2.8^{\circ}$ corresponding to a relative SD of $1.6 \%$. In general the trend is the same as for the simulated data as the estimated angles span the same interval.

\section{Comparison to standard TO}

The data acquired from the flow rig has also been beamformed and processed using the TO approach described in [27] using the dynamic apodization shown in Fig. 5. The relative SD and bias are shown in Fig. 16 for the optimized TO approach using 16 emissions.

The SD is only slightly lower for the DTO approach, but the bias is significantly lower. The bias for the angles for depths from 24 to $104 \mathrm{~mm}$ using 16 emission and TO is between -
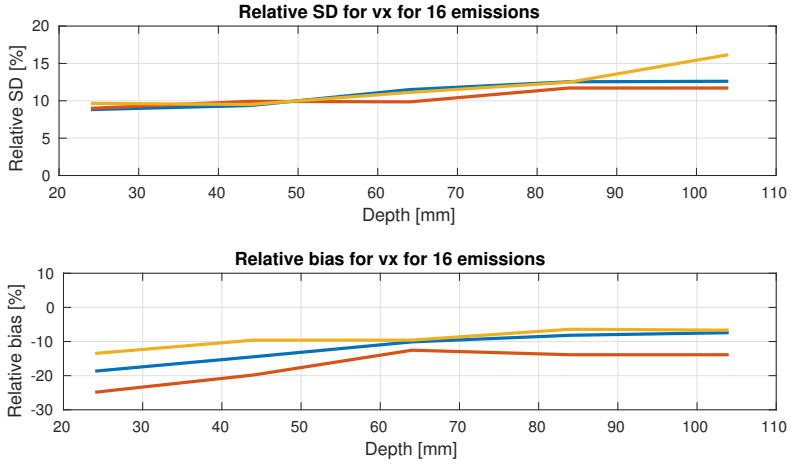

Fig. 16. Measured relative SD (top) and bias (bottom) using 16 emissions for the TO approach for the lateral velocity component.

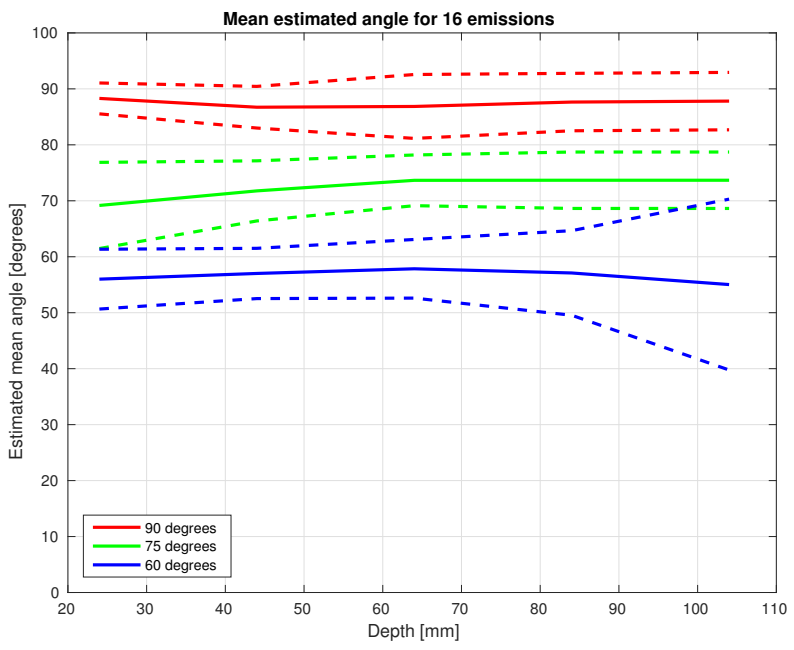

Fig. 17. Estimated angle \pm one standard deviation as a function depth and angle for measured data and 16 emissions using the TO approach.

$6.4 \%$ and $-24.9 \%$ with a mean for all situations of $-12.6 \%$. This should be compared with the mean bias for DTO of $-3.4 \%$.

The statistics for the estimated angles are shown in Fig. 17. The span of SDs for the angles for depths from 24 to $104 \mathrm{~mm}$ using 16 emissions is between $15.3^{\circ}$ and $2.8^{\circ}$ with a mean SD for all situations of $6.5^{\circ}$. This should be compared with $2.8^{\circ}$ for DTO.

\section{Deep vessel imaging}

The previous investigations were performed down to 104 $\mathrm{mm}$, but deeper lying vessels are often of interest. For these the active receive aperture cannot be expanded more, and the lateral oscillation period will therefore increase with depth, thus, reducing the resolution as in e.g. phased array imaging. To quantify this, experiments have been conducted at depths of 126 to $156 \mathrm{~mm}$ for the three angles. The transmit focus was maintained at $300 \mathrm{~mm}$ and $f_{p r f}$ was lowered to $2 \mathrm{kHz}$. The maximum depth of $156 \mathrm{~mm}$ was determined by the size of the mechanical transducer holder.

An example of profiles estimated at the maximum depth for a $90^{\circ}$ beam-to-flow angle is shown in Fig. 18. Thirty-two emissions were used, and the bias was $-8.5 \%$ for a SD of 

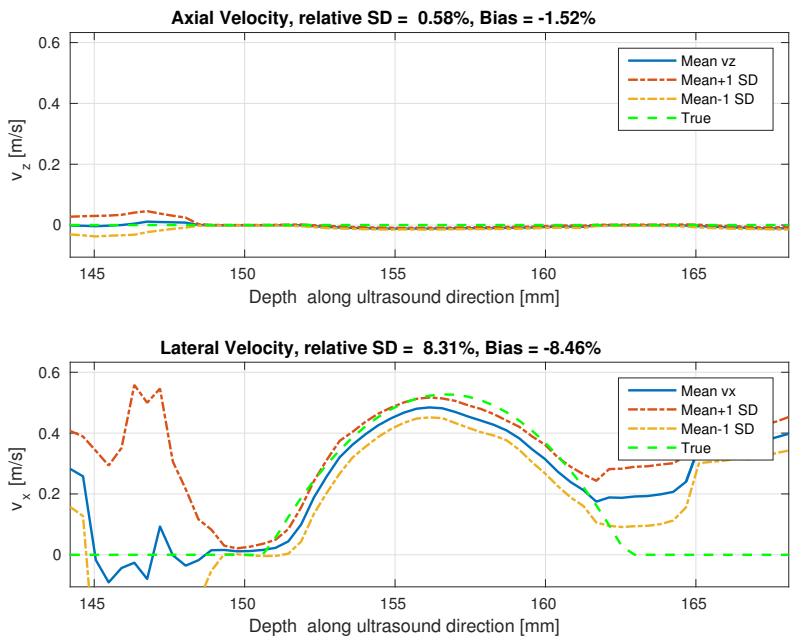

Fig. 18. Mean velocity profile for measured parabolic flow at a vessel depth of $156 \mathrm{~mm}$ and using 32 emissions for the estimation.
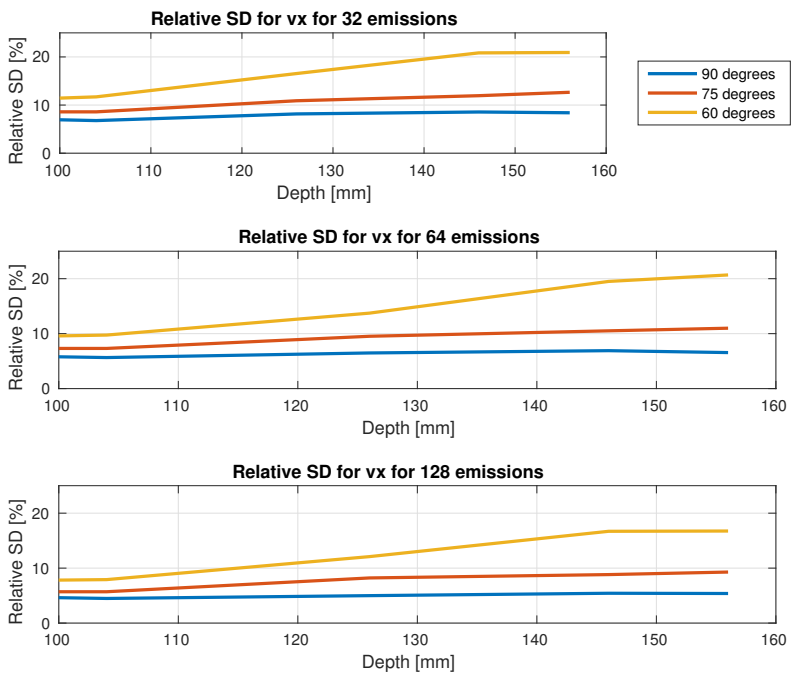

Fig. 19. Measured relative SD as a function depth and angle for 32 (top), 64 (middle), and 128 emissions (bottom).

$8.3 \%$, and the angle was found to be $91.4^{\circ}$ with a SD of $0.6^{\circ}$.

Larger depths need more emissions to attain the same performance due to the loss of energy, and Fig. 19 shows the SD for larger depths using 32, 64, and 128 emissions. For 32 emissions the bias ranges from $1.8 \%$ to $-10.3 \%$ with a mean of $-4.9 \%$ for all depths from 24 to $156 \mathrm{~mm}$.

A larger amount of emissions will lower the frame rate to an unacceptable level, but a duplex sequence can be used where the velocity vectors are found only along one line. This could give a quantitative replacement for spectral Doppler investigations, as described below for pulsating flow.

\section{E. Pulsating flow measurements}

The results shown above are for stationary, parabolic flow, where it is possible to average over long times to decrease the standard deviation. This is unrealistic in a clinical setting due to the acceleration of the flow. The velocity can, thus,

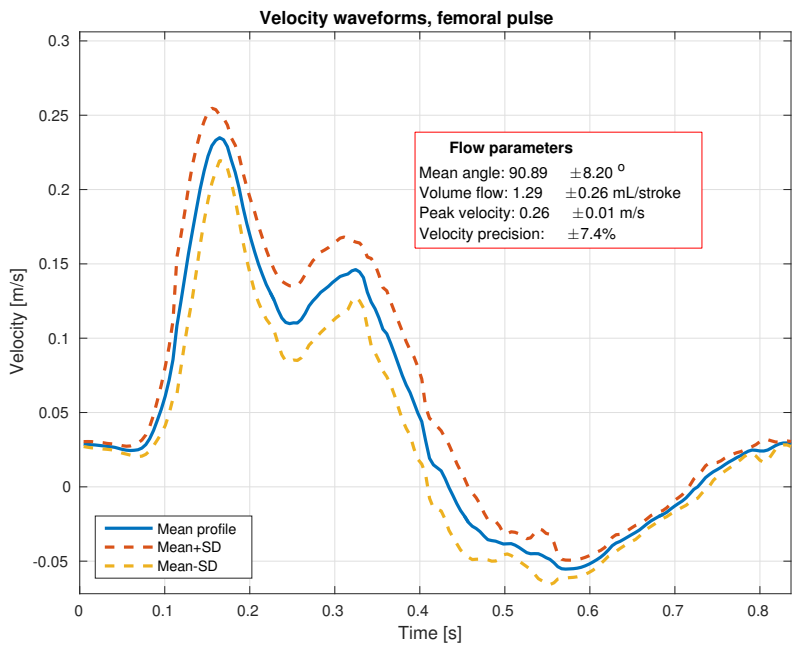

Fig. 20. Mean velocity at vessel center after automatic alignment for all 15 pulsation cycles along with estimated angle, stroke volume, and their respective precisions.

only be considered constant over a short time interval, and this will affect the accuracy. Data has therefore been measured for pulsating flow over 15 cardiac cycles for a femoral artery waveform. The profiles are estimated as a function of time and space in a single direction. The mean and SD was found over the cardiac cycle as shown in Fig. 20. The velocity at the center of the vessel was found using 128 emissions and the depth of the vessel center was $38.2 \mathrm{~mm}$. The waveforms for the individual heart cycles were aligned using crosscorrelation, and the velocity samples were aligned through spline interpolation.

The relative mean SD after alignment is $0.017 \mathrm{~m} / \mathrm{s}$ across the pulsation cycle. The peak velocity was $0.26 \mathrm{~m} / \mathrm{s}$ yielding a relative SD of $7.4 \%$ compared to the peak value or $0.87 \%$ compared to the peak detectable velocity. The peak velocity was found to $0.26 \mathrm{~m} / \mathrm{s}$ with an $\mathrm{SD}$ of $0.01 \mathrm{~m} / \mathrm{s}$ across heart beats. From this continuous data the stroke volume was found to be $1.29 \pm 0.26 \mathrm{~mL} /$ stroke (true value of pump: 1.15 $\mathrm{mL} /$ stroke, accuracy $3 \%, \pm 0.035 \mathrm{~mL} / \mathrm{stroke}$ ).

\section{Discussion AND CONCLUSION}

A new method for making TO vector flow estimation selfcalibrating has been suggested. The technique estimates the lateral oscillation period from the actual measured data and uses this in the velocity estimation. This makes it possible to have any apodization waveform and lower F-number focusing without having to predict the lateral oscillation period, and this maintains a low bias of the estimates for different depths. The approach also makes it possible to average over the lateral signal to decrease variance.

The fields used in TO imaging can be more narrowly focused using the new method. In previous approaches the transmit focus was kept high with an F-number of 5 to make focusing easier and obtain a one-sided spectrum. Now the Fnumber in receive can be lowered from 2 to 0.5 and thereby increase the lateral resolution. 
The self-calibrating feature of DTO was demonstrated for depths from 24 to $104 \mathrm{~mm}$ and for angles from $60^{\circ}$ to $90^{\circ}$. For 16 emissions the angle was found with an average SD of $2.8^{\circ}$. The relative SD could for angles above $60^{\circ}$ be maintained below $10 \%$ for the lateral velocity component with a slight increase in SD for decreasing angle. The SD was also roughly constant for all depths with a slightly increase at higher depths; probably due to a decrease in SNR. The SD for the lateral component is roughly 3 times that for the axial component. Performance could be slightly increased by increasing the number of emissions used for one estimate at a penalty in frame rate. Usable estimates with SDs around 10-15\% were obtained even for 8 emissions indicating that fast VFI can be made. Compared to optimized TO, the new approach gives a decrease in mean bias from $-12.6 \%$ to $-3.4 \%$. A similar performance gain is seen for the angle estimation.

VFI for deeper lying vessels from 126 to $156 \mathrm{~mm}$ could also be attained with a bias maintained below $10 \%$ and a slightly increased SD. This could be compensated for by increasing the number of emissions to yield quantitative velocity estimates at a reduced frame rate, or in a duplex sequence for finding velocity indices that do not have to be angle corrected.

A pulsating flow example showed that the continuous duplex data can be used for yielding quantitative flow measures and can potentially replace angle-corrected estimates based on spectral velocity estimation. From fifteen heart beats peak velocity, volume flow, beam-to-flow angle, and their precisions can be determined at any angle making estimation faster and less error prone than estimates based on the spectrum. This approach can also handle the situation with a time varying angle over the cardiac cycle, which is typical for more complex flow in tortuous or stenosed vessels. The angle variation is also a direct indication of turbulence and disturbed flow.

The bias is nearly always negative for the measurements conducted here. This is probably due to the averaging of the velocity estimates across the width of the point spread function. This will inevitably lead to a lower velocity, and the peak velocity in a vessel is difficult to estimate precisely. The bias is, however, small (around $-5 \%$ across all depths) and could be scaled out, but there are different biases (range from $1.8 \%$ to $-10.3 \%$ ) as it depends on depth and flow angle making a fixed compensation of little value.

The drawback of DTO is the increased beamforming and processing to perform. The number of parallel beams is increased by the number of samples in the directional signal, and this can be around 10 to 20 times that of TO beamforming. Here 80 samples are beamformed per directional line. Modern scanners can, however, make several beams in parallel up to 64, and this matches the demand for DTO.

The approach can also be employed for three-dimensional velocity estimation. This necessitates the use of a twodimensional transducer and beamforming in two orthogonal planes. The TO vector velocity estimation for 3-D imaging is described in [42], [43]. Here five lines are beamformed in parallel to find the velocity estimates for all three velocity components $v_{x}, v_{y}$, and $v_{z}$. The normal in-phase and quadrature beamforming is here replaced by a transverse beamforming orthogonal to the ultrasound beam direction in the $x-z$ plane and in the $y-z$ plane. The estimation scheme described above can then be employed.

\section{ACKNOWLEDGEMENT}

This work was supported by grant 82-2012-4 from the Danish Advanced Technology Foundation and by B-K Ultrasound Aps, Herlev, Denmark.

\section{REFERENCES}

[1] G. E. Trahey, J. W. Allison, and O. T. von Ramm, "Angle independent ultrasonic detection of blood flow," IEEE Trans. Biomed. Eng., vol. BME-34, no. 12, pp. 965-967, 1987.

[2] M. D. Fox, "Multiple crossed-beam ultrasound Doppler velocimetry," IEEE Trans. Son. Ultrason., vol. SU-25, pp. 281-286, 1978.

[3] B. Dunmire, K. W. Beach, K.-H. Labs., M. Plett, and D. E. Strandness, "Cross-beam vector Doppler ultrasound for angle independent velocity measurements," Ultrasound Med. Biol., vol. 26, pp. 1213-1235, 2000.

[4] O. Bonnefous, "Measurement of the complete (3D) velocity vector of blood flows," in Proc. IEEE Ultrason. Symp., 1988, pp. 795-799.

[5] J. A. Jensen, "Directional velocity estimation using focusing along the flow direction: I: Theory and simulation," IEEE Trans. Ultrason., Ferroelec., Freq. Contr., vol. 50, pp. 857-872, 2003.

[6] S. I. Nikolov and J. A. Jensen, "In-vivo synthetic aperture flow imaging in medical ultrasound," IEEE Trans. Ultrason., Ferroelec., Freq. Contr., vol. 50 , no. 7, pp. 848-856, 2003.

[7] J. Udesen, F. Gran, K. L. Hansen, J. A. Jensen, C. Thomsen, and M. B. Nielsen, "High frame-rate blood vector velocity imaging using plane waves: simulations and preliminary experiments," IEEE Trans. Ultrason., Ferroelec., Freq. Contr., vol. 55, no. 8, pp. 1729-1743, 2008.

[8] J. Bercoff, G. Montaldo, T. Loupas, D. Savery, F. Meziere, M. Fink, and M. Tanter, "Ultrafast compound Doppler imaging: providing full blood flow characterization," IEEE Trans. Ultrason., Ferroelec., Freq. Contr., vol. 58, no. 1, pp. 134-147, January 2011.

[9] I. K. Ekroll, A. Swillens, P. Segers, T. Dahl, H. Torp, and L. Lovstakken, "Simultaneous quantification of flow and tissue velocities based on multi-angle plane wave imaging," IEEE Trans. Ultrason., Ferroelec., Freq. Contr., vol. 60, no. 4, pp. 727-738, 2013.

[10] A. Pastorelli, G. Torricelli, M. Scabia, E. Biagi, and L. Masotti, "A real-time 2-D vector Doppler system for clinical experimentation," IEEE Trans. Med. Imag., vol. 27, no. 10, pp. 1515-1524, 2008.

[11] A. Swillens, P. Segers, H. Torp, and L. Løvstakken, "Two-dimensional blood velocity estimation with ultrasound: speckle tracking versus crossed-beam vector Doppler based on flow simulations in a carotid bifurcation model," IEEE Trans. Ultrason., Ferroelec., Freq. Contr., vol. 57, no. 2, pp. 327-339, 2010.

[12] S. Fadnes, I. K. Ekroll, S. A. Nyrnes, H. Torp, and L. Løvstakken, "Robust angle-independent blood velocity estimation based on dualangle plane wave imaging," IEEE Trans. Ultrason., Ferroelec., Freq. Contr., vol. 62, no. 10, pp. 1757-1767, October 2015.

[13] J. Provost, C. Papadacci, C. Demene, J.-L. Gennisson, M. Tanter, and M. Pernot, "3-d ultrafast Doppler imaging applied to the noninvasive mapping of blood vessels in vivo," IEEE Trans. Ultrason., Ferroelec., Freq. Contr., vol. 62, no. 8, pp. 1467-1472, 2015.

[14] J. A. Jensen, S. I. Nikolov, A. Yu, and D. Garcia, "Ultrasound vector flow imaging I: Sequential systems," IEEE Trans. Ultrason., Ferroelec., Freq. Contr., vol. 63, no. 11, pp. 1704-1721, 2016.

[15] - "Ultrasound vector flow imaging II: Parallel systems," IEEE Trans. Ultrason., Ferroelec., Freq. Contr., vol. 63, no. 11, pp. 1722-1732, 2016.

[16] J. A. Jensen and P. Munk, "A new method for estimation of velocity vectors," IEEE Trans. Ultrason., Ferroelec., Freq. Contr., vol. 45, no. 3, pp. 837-851, 1998.

[17] J. A. Jensen, "Apparatus and method for determining movements and velocities of moving objects," International patent PCT/DK97/00287, 1996.

[18] _ - "A new estimator for vector velocity estimation," IEEE Trans. Ultrason., Ferroelec., Freq. Contr., vol. 48, no. 4, pp. 886-894, 2001.

[19] M. E. Anderson, "Multi-dimensional velocity estimation with ultrasound using spatial quadrature," IEEE Trans. Ultrason., Ferroelec., Freq. Contr., vol. 45, pp. 852-861, 1998.

[20] C. Sumi, "Displacement vector measurement using instantaneous ultrasound signal phase - multidimensional autocorrelation and Doppler methods," IEEE Trans. Ultrason., Ferroelec., Freq. Contr., vol. 55, no. 1 , pp. 24-43, 2008. 
[21] H. Liebgott, J. Wilhjelm, J. A. Jensen, D. Vray, and P. Delachartre "PSF dedicated to estimation of displacement vectors for tissue elasticity imaging with ultrasound,' IEEE Trans. Ultrason., Ferroelec., Freq. Contr., vol. 54, no. 4, pp. 746-756, 2007.

[22] H. Liebgott, A. Basarab, P. Gueth, C. Cachard, and P. Delachartre, "Lateral RF image synthesis using a synthetic aperture imaging technique." IEEE Trans. Ultrason., Ferroelec., Freq. Contr., vol. 55, no. 9, pp. 20972103, 2008.

[23] A. Basarab, P. Gueth, H. Liebgott, and P. Delachartre, "Phase-based block matching applied to motion estimation with unconventional beamforming strategies," IEEE Trans. Ultrason., Ferroelec., Freq. Contr., vol. 56, no. 5, pp. 945-957, 2009.

[24] M. Lenge, A. Ramalli, P. Tortoli, C. Cachard, and H. Liebgott, "Planewave transverse oscillation for high-frame-rate 2-D vector flow imaging," IEEE Trans. Ultrason., Ferroelec., Freq. Contr., vol. 62, no. 12, pp. 2126-2137, December 2015.

[25] J. A. Jensen, Estimation of Blood Velocities Using Ultrasound: A Signal Processing Approach. New York: Cambridge University Press, 1996.

[26] J. W. Goodman, Introduction to Fourier optics, 2nd ed. New York: McGraw Hill Inc., 1996.

[27] J. A. Jensen, A. H. Brandt, and M. B. Nielsen, "Convex array vector velocity imaging using transverse oscillation and its optimization," IEEE Trans. Ultrason., Ferroelec., Freq. Contr., vol. 62, no. 12, pp. $2043-$ 2053, 2015

[28] J. A. Jensen, "Improved vector velocity estimation using directional transverse oscillation," in Proc. IEEE Ultrason. Symp., October 2015, pp. $1-4$.

[29] J. A. Jensen and S. I. Nikolov, "Directional synthetic aperture flow imaging," IEEE Trans. Ultrason., Ferroelec., Freq. Contr., vol. 51, pp. 1107-1118, 2004.

[30] J. Kortbek and J. A. Jensen, "Estimation of velocity vector angles using the directional cross-correlation method," IEEE Trans. Ultrason., Ferroelec., Freq. Contr., vol. 53, pp. 2036-2049, 2006.

[31] J. A. Jensen and N. Oddershede, "Estimation of velocity vectors in synthetic aperture ultrasound imaging," IEEE Trans. Med. Imag., vol. 25, pp. 1637-1644, 2006.

[32] T. Loupas, J. T. Powers, and R. W. Gill, "An axial velocity estimator for ultrasound blood flow imaging, based on a full evaluation of the Doppler equation by means of a two-dimensional autocorrelation approach," IEEE Trans. Ultrason., Ferroelec., Freq. Contr., vol. 42, pp. 672-688, 1995.

[33] C. Kasai, K. Namekawa, A. Koyano, and R. Omoto, "Real-Time TwoDimensional Blood Flow Imaging using an Autocorrelation Technique," IEEE Trans. Son. Ultrason., vol. 32, no. 3, pp. 458-463, 1985.

[34] T. Loupas and R. W. Gill, "Multifrequency Doppler: Improving the quality of spectral estimation by making full use of the information present in the backscattered rf echoes," IEEE Trans. Ultrason., Ferroelec., Freq. Contr., vol. 41, pp. 522-531, 1994.

[35] T. Loupas, R. B. Peterson, and R. W. Gill, "Experimental evaluation of velocity and power estimation for blood flow imaging, by means of a two-dimensional autocorrelation approach," IEEE Trans. Ultrason., Ferroelec., Freq. Contr., vol. 42, pp. 689-699, 1995.

[36] J. A. Jensen, H. Holten-Lund, R. T. Nilsson, M. Hansen, U. D. Larsen, R. P. Domsten, B. G. Tomov, M. B. Stuart, S. I. Nikolov, M. J. Pihl, Y. Du, J. H. Rasmussen, and M. F. Rasmussen, "SARUS: A synthetic aperture real-time ultrasound system," IEEE Trans. Ultrason., Ferroelec., Freq. Contr., vol. 60, no. 9, pp. 1838-1852, 2013.

[37] J. A. Jensen and N. B. Svendsen, "Calculation of pressure fields from arbitrarily shaped, apodized, and excited ultrasound transducers," IEEE Trans. Ultrason., Ferroelec., Freq. Contr., vol. 39, pp. 262-267, 1992.

[38] J. A. Jensen, "Field: A program for simulating ultrasound systems," Med. Biol. Eng. Comp., vol. 10th Nordic-Baltic Conference on Biomedical Imaging, Vol. 4, Supplement 1, Part 1, pp. 351-353, 1996.

[39] — " "A multi-threaded version of Field II," in Proc. IEEE Ultrason. Symp. IEEE, 2014, pp. 2229-2232.

[40] $\longrightarrow$, "Safety assessment of advanced imaging sequences, II: Simulations," IEEE Trans. Ultrason., Ferroelec., Freq. Contr., vol. 63, no. 1, pp. 120-127, 2016.

[41] J. M. Hansen, M. C. Hemmsen, and J. A. Jensen, "An object-oriented multi-threaded software beamformation toolbox," in Proc. SPIE Med. Imag., vol. 7968, March 2011, pp. 79680Y-1-79680Y-9.
[42] M. J. Pihl and J. A. Jensen, "A transverse oscillation approach for estimation of three-dimensional velocity vectors. Part I: Concept and simulation study," IEEE Trans. Ultrason., Ferroelec., Freq. Contr., vol. 61, pp. 1599-1607, 2014.

[43] M. J. Pihl, M. B. Stuart, B. G. Tomov, M. F. Rasmussen, and J. A. Jensen, "A transverse oscillation approach for estimation of threedimensional velocity vectors. Part II: Experimental validation," IEEE Trans. Ultrason., Ferroelec., Freq. Contr., vol. 51, no. 10, pp. 16081618,2014

\section{BIBLIOGRAPHIES}

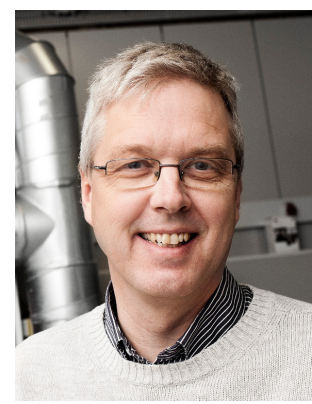

Jørgen Arendt Jensen earned his Master of Science in electrical engineering in 1985 and the $\mathrm{PhD}$ degree in 1989, both from the Technical University of Denmark. He received the Dr.Techn. degree from the university in 1996. He has since 1993 been full professor of Biomedical Signal Processing at the Technical University of Denmark at the Department of Electrical Engineering and head of Center for Fast Ultrasound Imaging since its inauguration in 1998. He has published more than 400 journal and conference papers on signal processing and medical ultrasound and the book "Estimation of Blood Velocities Using Ultrasound", Cambridge University Press in 1996. He is also the developer and maintainer of the Field II simulation program. He has been a visiting scientist at Duke University, Stanford University, and the University of Illinois at Urbana-Champaign. He was head of the Biomedical Engineering group from 2007 to 2010. In 2003 he was one of the founders of the biomedical engineering program in Medicine and Technology, which is a joint degree program between the Technical University of Denmark and the Faculty of Health and Medical Sciences at the University of Copenhagen. The degree is one of the most sought after engineering degrees in Denmark. He was chairman of the study board from 2003-2010 and adjunct professor at the University of Copenhagen from 2005-2010. He has given a number of short courses on simulation, synthetic aperture imaging, and flow estimation at international scientific conferences and teaches biomedical signal processing and medical imaging at the Technical University of Denmark. He has given more than 60 invited talks at international meetings, received several awards for his research, lately the Grand Solutions prize from the Danish Minister of Science. He is an IEEE Fellow since 2012. His research is centered around simulation of ultrasound imaging, synthetic aperture imaging, vector blood flow estimation, and construction of ultrasound research systems. 